\title{
New Pfaffian formulae for an overlap of multiple quasiparticle states
}

\author{
Makito $\mathrm{Oi}^{1, \mathrm{a}}$ and Takahiro Mizusaki ${ }^{1}$ \\ ${ }^{1}$ Institute of Natural Sciences, Senshu University, 3-8 Kanda-Jinbocho, Chiyoda-ku 101-8425, Tokyo, Japan
}

\begin{abstract}
The Pfaffian formulae recently derived by the authors are presented. They can be widely applied to evaluations of a variety of quantities, necessary in nuclear manybody theories, such as the generator coordinate method (GCM). As an example, an application to the energy expectation is presented.
\end{abstract}

\section{Introduction}

The superposition principle is the central concept in Quantum Mechanics. A given quantum state $\Psi$ can be expanded by an arbitrary complete set of basis. However, there are cases that one might want to approximate $\Psi$ by a single ansatz state rather than to expand rigorously with the infinite (or quite large) number of basis states. In general, complicated quantum states (such as interacting many-body states) tend to prevent us from obtaining an intuitive understanding of the physical meaning of the state if the rigorous expansion is applied. In such cases, it is often seen (like the Bardeen-CooperSchrieffer theory) that the single ansatz clarifies the intricacy coming from quantum entanglements although we have to admit that the ansatz is just an approximation.

The variational method is often found to be effective in finding such an ansatz. Once a variational state $\Psi_{0}$ is established, it can be regarded as a "classical" solution to the problem of interest. For a further accuracy, the obtained state needs to be corrected so as to take into account quantum fluctuations around the classical solution. Schematically, such a state with quantum fluctuations $\delta \Psi_{0}$ can be written as

$$
|\Psi\rangle=\left|\Psi_{0}\right\rangle+\left|\delta \Psi_{0}\right\rangle
$$

If the fluctuation has a small amplitude, it can be parametrised by a certain "small" quantity $\epsilon$ where the second order in $\epsilon$ and the higher orders can be neglected safely, or $\left\langle\delta \Psi_{0} \mid \delta \Psi_{0}\right\rangle /\left\langle\Psi_{0} \mid \Psi_{0}\right\rangle<<1$. The random phase approximation (RPA) corresponds to such a linearised theory in $\epsilon$ to deal with quantum fluctuation. However, there remain cases where $\delta \Psi_{0}$ has large-amplitude quantum fluctuations, or $\left\langle\delta \Psi_{0} \mid \delta \Psi_{0}\right\rangle /\left\langle\Psi_{0} \mid \Psi_{0}\right\rangle \simeq 1$. In this case, the linear approximation in $\epsilon$ cannot be justified. For example, such a large-amplitude fluctuation is seen in a case when $\delta \Psi_{0}$ is obtained by a zero-mode shift of the original variational state $\Psi_{0}$ through a relevant symmetry operation $\hat{R}(g)$. In this case, $\delta \Psi_{0}$ is labelled by an element $g$ in the symmetry group $G$, as $\left|\delta \Psi_{0}\right\rangle \rightarrow \hat{R}(g)\left|\Psi_{0}\right\rangle=|\Psi(g)\rangle$. Because all the zero-mode states are energetically degenerate, it is natural to express $\delta \Psi_{0}$ as a superposition $\left|\delta \Psi_{0}\right\rangle=$ $\sum_{g} C_{g}|\Psi(g)\rangle$. If the group $G$ corresponds to a continuous symmetry group, such as the Lie group, then

\footnotetext{
am.oi@isc.senshu-u.ac.jp
}

This is an Open Access article distributed under the terms of the Creative Commons Attribution License 2.0, which permits unrestricted use, distribution, and reproduction in any medium, provided the original work is properly cited. 
the summation in $g$ is replaced with the integration over $g$. Considering the original variational state $\Psi_{0}$ as the identity element in $G$, the quantum state of interest $\Psi$ can be expressed as

$$
|\Psi\rangle=\int f(g)|\Psi(g)\rangle d g
$$

This way of superposition is nothing but the generator coordinate method (GCM) [1], and it is possible to interpret the superposition as the restoration of the symmetry broken by the variational state $\Psi_{0}$, which is similar to the Nambu-Goldstone mode in Quantum Field Theory.

The function $f(g)$ which corresponds to the expansion coefficients $C_{g}$ is determined in the variational way. That is,

$$
\delta \frac{\langle\Psi|\hat{H}| \Psi\rangle}{\langle\Psi \mid \Psi\rangle}=0,
$$

where the Hamiltonian is denoted as $\hat{H}$. This variational equation gives rise to the so-called the HillWheeler equation, which consists of overlaps of operators with respect to the mean-field state as well as its zero modes $\Psi(g)$. One of such overlaps is the norm overlap, which is given as

$$
N\left(g, g^{\prime}\right)=\left\langle\Psi(g) \mid \Psi\left(g^{\prime}\right)\right\rangle
$$

Evaluation of this quantity had been a perennial problem, especially for interacting many-body states in the presence of the pairing correlations.

\section{The Hartree-Fock-Bogoliubov ansatz}

As a mean-field description of nuclear many-body states, the Hartree-Fock-Bogoliubov (HFB) ansatz,

$$
\left|\Psi_{0}\right\rangle=\exp \left(\frac{1}{2} \sum_{i j} M_{i j} c_{i}^{\dagger} c_{j}^{\dagger}\right)|0\rangle,
$$

has been building up positive reputations since its first application to nuclear systems soon after the discovery of the BCS (Bardeen-Cooper-Schrieffer) ansatz in the theory of superconductivity. In the above expression, $c^{\dagger}(c)$ is the creation (annihilation) operator of a nucleon. The vacuum associated with the constituent nucleons is denoted as $|0\rangle$, hence $c_{i}|0\rangle=0$. $M$ is a complex matrix related to the canonical transformation between the real and quasi particles (The Bogoliubov transformation).

The HFB state $\Psi_{0}$ breaks the rotational symmetry $(\mathrm{SO}(3))$ and the Gauge symmetry (U(1)) by allowing nuclear deformation and the Cooper-pair condensation. Large-amplitude fluctuations are thus induced by the relevant symmetry operations, $|\Psi(\Omega)\rangle=\hat{R}(\Omega)\left|\Psi_{0}\right\rangle$. If $\Omega$ is a continuous parameter and the operation $\hat{R}$ follows the Lie-group structure, the unitarity condition can be imposed on the operation, hence $\hat{R}^{\dagger} \hat{R}=\hat{R} \hat{R}^{\dagger}=\hat{I}$, where $\hat{I}$ means the identity operation. With the help of the BakerHaussdorf formula and a matrix representation of $\hat{R}$, one can demonstrate that the zero-mode $|\Psi(\Omega)\rangle$ is also an HFB-type state,

$$
|\Psi(\Omega)\rangle=\exp \left(\frac{1}{2} \sum_{i j} M(\Omega)_{i j} c_{i}^{\dagger} c_{j}^{\dagger}\right)|0\rangle,
$$

where $M(\Omega)=R(\Omega) M R^{T}(\Omega)$. 


\section{Analytical formulae for HFB overlaps}

Evaluation of an overlap between different HFB states had been a perennial problem even though several analytical formulae were discovered, such as the Onishi formula [2],

$$
\left\langle\Psi(\Omega) \mid \Psi\left(\Omega^{\prime}\right)\right\rangle=\sqrt{\operatorname{det} P\left(\Omega, \Omega^{\prime}\right)},
$$

where $P$ is a complex-valued matrix connected closely to the Bogoliubov transformation. $\operatorname{det} P\left(\Omega, \Omega^{\prime}\right)$ is a complex function of $\Omega$ and $\Omega^{\prime}$, so that its square root demands a proper treatment to set up the Riemann surface in order to establish the determinant as a single-valued function. This treatment brings a complication in the evaluation of the norm overlap, which has been a nuisance for long time [3].

A breakthrough came recently to remove all the mathematical difficulties related to the square root operation (sign problem) in the know formulae, which was brought by Robledo with the Pfaffian [4],

$$
N\left(\Omega, \Omega^{\prime}\right)=\operatorname{Pf}\left(\mathbb{M}\left(\Omega, \Omega^{\prime}\right)\right),
$$

where $\mathbb{M}$ is a bipartite extension of $M$ in the HFB ansatz. The Pfaffian formula was derived with the help of Fermion coherent state and Grassmann algebra. Both of them had been recognized as the most foreign to many nuclear-structure physicists.

\section{The Pfaffian formulae}

Many theoretical works followed Robledo's pioneering work [5-9] in order to extend Robledo's formula to multiple quasiparticle excited states. The extension essentially corresponds to finding a formula to evaluate a quantity such as $\left\langle\Psi(\Omega)\left|c_{i}^{\dagger} c_{j}^{\dagger} \cdots c_{k}^{\dagger} c_{l} c_{m} \cdots c_{n}\right| \Psi\left(\Omega^{\prime}\right)\right\rangle$. It has been known that the generalised Wick's theorem can deal with this type of quantities. Unfortunately, Wick's theorem shows us only a guidance for the evaluation, but does not give us an explicit formula. As a result, people relying on Wick's theorem need to write down the above quantity with the contractions in an ad hoc manner and the result tends to be quite lengthy like a telephone book. Thanks to the recent progress in the application of the Pfaffian, systematic and compact formulae were finally obtained.

With the aim of demonstrating the power of the Pfaffian formulae, let us try to evaluate an expectation value of the general Hamiltonian consisting of the one-body and two-body parts, that is,

$$
\hat{H}=\sum_{i j} t_{i j} c_{i}^{\dagger} c_{j}+\frac{1}{2} \sum_{i j k l} v_{i j k l} c_{i}^{\dagger} c_{j}^{\dagger} c_{l} c_{k},
$$

with respect to the HFB state $\Psi_{0}$, as a simple example. (An extension to the matrix elements, that is, an overlap with respect to two different HFB states, $\Psi(\Omega)$ and $\Psi\left(\Omega^{\prime}\right)$ can be carried out in a straightforward manner. See [10] for details.)

\subsection{An application of the Pfaffian formulae: Calculation of $\langle\hat{H}\rangle$}

Before starting the calculation, one should know that we need to pay attention to the ordering of products of the annihilation and creation operators. In usual Wick's theorem, the so-called "normal ordering" is introduced, which is a product where the annihilation operators are placed in the right half end of the product, such as $c^{\dagger} \cdots c^{\dagger} c \cdots c$. If the expectation value of the product is taken with respect to the vacuum to the $\left(c, c^{\dagger}\right)$ basis, then all the normal ordered products vanish. Wick's theorem (as well as the generalised Wick's theorem) says that any product of the creation and annihilation 
operators can be expressed as a sum of normal ordered products and the contractions. Thanks to this property of the normal ordered products, the expectation value (and the matrix element) of the product can be simplified greatly in terms of the algebraic expression with the contractions.

In the derivations of the Pfaffian formulae, we rely on the Fermion coherent state $|\xi\rangle$, which is the eigenstate of the annihilation operators, hence $c_{i}|\xi\rangle=\xi_{i}|\xi\rangle$. Because of this property, it is convenient to reverse the normal ordering, that is, $c \cdots c c^{\dagger} \cdots c^{\dagger}$ for the calculation of expectation values in terms of the Pfaffian. Therefore, we should begin with making the "reversal ordering" of the operators.

Firstly, the one-body operator in Eq.(9) can be easily evaluated as a special case of the formula derived in Ref.[7], where one-quasiparticle excited states are expressed as $\left|\Psi_{0, i}\right\rangle=\beta_{i}^{\dagger}\left|\Psi_{0}\right\rangle=c_{i}^{\dagger}\left|\Psi_{0}\right\rangle$. Consequently, we obtain

$$
\left\langle\Psi_{0}\left|c_{i}^{\dagger} c_{j}\right| \Psi_{0}\right\rangle=\operatorname{Pf}(\mathbb{X})\left(\delta_{i j}-\mathbb{X}_{i \bar{j}}^{-1}\right)
$$

Secondly, the two-body operator in Eq.(9) contains a product $c_{i}^{\dagger} c_{j}^{\dagger} c_{l} c_{k}$, which needs to be rearranged to a "reversal order" as

$$
c_{i}^{\dagger} c_{j}^{\dagger} c_{l} c_{k}=c_{l} c_{k} c_{i}^{\dagger} c_{j}^{\dagger}+\left[c_{i}^{\dagger} c_{j}^{\dagger}, c_{l} c_{k}\right]
$$

where

$$
\left[c_{i}^{\dagger} c_{j}^{\dagger}, c_{l} c_{k}\right]=\delta_{j k} a_{l} a_{i}^{\dagger}+\delta_{i l} a_{k} a_{j}^{\dagger}-\delta_{i k} a_{l} a_{j}^{\dagger}-\delta_{j l} a_{k} a_{i}^{\dagger}+\delta_{j l} \delta_{i k}-\delta_{j k} \delta_{i l} .
$$

The expectation value of the first term $\left\langle\Psi_{0}\left|c_{l} c_{k} c_{i}^{\dagger} c_{j}^{\dagger}\right| \Psi_{0}\right\rangle$ in the right-hand side of Eq.(11) can be evaluated with an aid of the formula derived in $[8]$ to be $\operatorname{Pf}(\mathbb{X}) \operatorname{Pf}\left(\mathbb{X}_{\{j, i, \bar{k}, \bar{l}\}}^{-1}\right)$, which was demonstrated explicitly as an example in Eq.(46) of Ref.[8]. See Eq.(44) in Ref.[8] for the definition of $\mathbb{X}^{-1}$, which can be regarded as the "antisymmetrised" bipartite density matrix. The expectation value of the second term in Eq.(11), or Eq.(12), can be evaluated easily with the help of the formula derived in Ref.[7] (for the first three terms of one-body parts in Eq.(12)) and Robledo's original Pfaffian formula [4] (for the last two terms in Eq.(12). By combining the above results, one can evaluate the energy expectation value $\left\langle\Psi_{0}|\hat{H}| \Psi_{0}\right\rangle$ in the Pfaffian form. This expression can be constructed simply with an aid of the known formulae recently derived by the authors (and others). Although the expression contains nothing new, it should be so useful in practical computations that it is worth trying to write it down individually.

\section{Summary}

We recently derived the Pfaffian formulae. The formulae improve what one has been struggling to evalute with Wick's theorem. The formulae found recently by the authors (and others) can be applied not only to overlaps but also expectation values and matrix elements of many-body operators.

\section{References}

[1] P. Ring and P. Schuck, The Nuclear Many-body Problem (Springer, Berlin, 1980) pp.398-437.

[2] N. Onishi and S. Yoshida, Nucl. Phys. 80367 (1966).

[3] M. Oi and N. Tajima, Phys. Lett. B 606, 43 (2005).

[4] L. M. Robledo, PHys. Rev. C 79, 021302(R) (2009).

[5] G. Bertsch and L. M. Robledo, Phys. Rev. Lett. 108, 042505 (2012).

[6] B. Avez and M. Bender, Phys. Rev. C 85, 034325 (2012).

[7] M. Oi and T. Mizusaki, Phys. Lett. B 707, 305 (2012).

[8] T. Mizusaki and M. Oi, Phys. Lett. B 715219 (2012).

[9] T. Mizusaki, M. Oi, F.-Q. Chen, Y. Sun, Phys. Lett. B 725175 (2013).

[10] K. Tanabe, K. Enami, N. Yoshinaga, Phys. Rev. C 592494 (1999). 